Research Article

\title{
Bioinspired Feathered Flapping Wing UAV Design for Operation in Gusty Environment
}

\author{
S. H. Abbasi (iD, A. Mahmood, and Abdul Khaliq \\ Department of Electrical and Computer Engineering, SS CASE IT, Islamabad, Pakistan \\ Correspondence should be addressed to S. H. Abbasi; abbasi882@gmail.com
}

Received 29 July 2021; Accepted 27 August 2021; Published 13 September 2021

Academic Editor: L. Fortuna

Copyright (c) 2021 S. H. Abbasi et al. This is an open access article distributed under the Creative Commons Attribution License, which permits unrestricted use, distribution, and reproduction in any medium, provided the original work is properly cited.

\begin{abstract}
The flight of unmanned aerial vehicles (UAVs) has numerous associated challenges. Small size is the major reason of their sensitivity towards turbulence restraining them from stable flight. Turbulence alleviation strategies of birds have been explored in recent past in detail to sort out this issue. Besides using primary and secondary feathers, birds also utilize covert feathers deflection to mitigate turbulence. Motivated from covert feathers of birds, this paper presents biologically inspired gust mitigation system (GMS) for a flapping wing UAV (FUAV). GMS consists of electromechanical (EM) covert feathers that sense the incoming gust and mitigate it through deflection of these feathers. A multibody model of gust-mitigating FUAV is developed appending models of the subsystems including rigid body, propulsion system, flapping mechanism, and GMS-installed wings using bond graph modeling approach. FUAV without GMS and FUAV with the proposed GMS integrated in it are simulated in the presence of vertical gust, and results' comparison proves the efficacy of the proposed design. Furthermore, agreement between experimental results and present results validates the accuracy of the proposed design and developed model.
\end{abstract}

\section{Introduction}

Attitude control is a serious concern for UAVs operating in the atmospheric boundary layer (ABL). This ABL region is best suitable for UAV applications in Intelligence, Surveillance, and Reconnaissance (ISR) missions. ABL, however, is considered as highly turbulent as shown in Figure $1[1,2]$.

The aircrafts flying in this region experience abrupt and severe gusts that can cause fast disturbances in orientation, attitude, position, and speed. The interaction of airflow with ground obstacles changes due to various factors comprising size, density, shape, and permeability. The Department of Land Resources has investigated the turbulence modes present in forested regions. It was revealed that the forests are the key turbulence source due to their patchy surface. In addition, the trees also disturb the profile of the turbulent flow because of drag in close vicinity to the tree line [3-5]. The Matterhorn, Switzerland, shows turbulence in mountainous ranges by the snow that is trapped within the wind shear in vicinity of the summit [6].
Significant degradation in performance of UAVs has been observed in the presence of high gusts and intense turbulence [7-12]. Furthermore, one of the major reasons of UAV loss at low altitudes operations is adverse winds [13]. Researches have indicated the loss of these UAVs is the result of lacking feedback from the sensors and huge time delay in transmission of data from the UAV to the pilot's control system [14]. Therefore, to continue stable UAV operation in these turbulent regions, a GMS is inevitable to optimize the aircraft's stability while decreasing danger of crashing.

After the Wright brothers' first journey, numerous flight controllers have been designed to tackle these turbulent airflows. The first ever GMS was developed in 1914 [15], after that, many attempts have been made to design an autonomous GMS, including study by the Bristol Company 1949 [16], Douglas Firm 1950 [17], and NACA 1952 [18, 19].

The use of the Global Positioning System (GPS) and autopilot modules have reduced pilot workload and improved safety while operating in gusty weather. Each subsystem is interlinked with the UAV's central flight computer and empowers the flight crew to closely assess the desired 


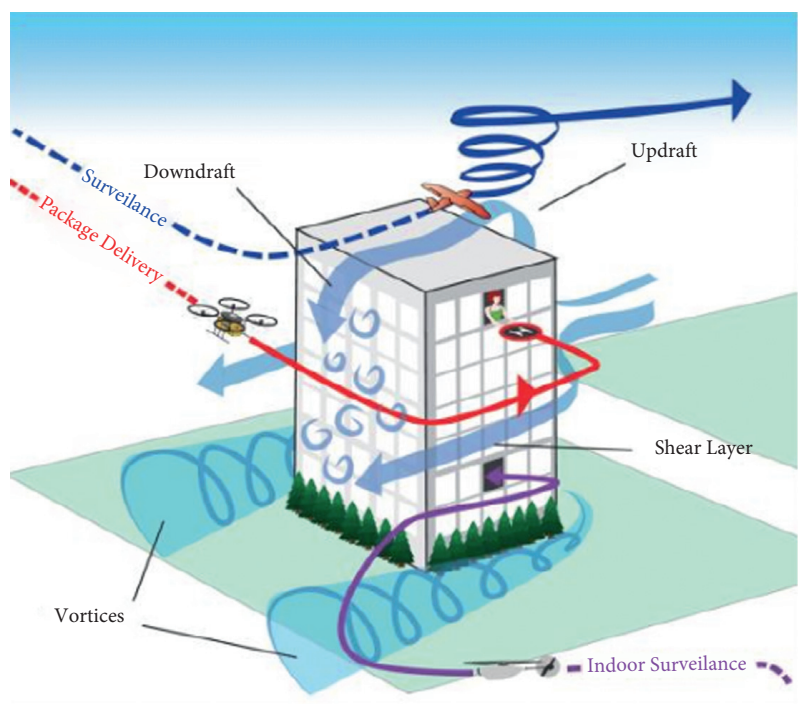

FIgURE 1: Airflow around buildings [1].

flight path. Resultantly, preemptive actions can be taken prior to facing bad weather and turbulent airflow to reduce gusting intensity, resulting in precautionary modifications in heading and altitude [20].

Several other designs have been made to advance UAV avionics to attain higher performance in the turbulent airflows. Study in [21] presented the advanced avionics for UAVs that can achieve stabilization performance similar to large-sized aircrafts. A Micro Architecture and Control (MARC) avionics design is developed having substantial improvements in weight constraints and power consumption of UAVs.

In addition to the aforementioned design efforts, during the last decade bioinspiration has also emerged as a breakthrough for solutions to many impending engineering problems. Researchers have carried out in-depth study of birds that fly successfully close to the ground as well as in forests in turbulent conditions. Biologically inspired UAVs are not new [22-25]. Several bioinspired flow sensors have been developed to date to alleviate turbulence. These include Particle Image Velocimetry (PIV), Light Detection and Ranging (LiDAR), Laser Doppler Anemometry (LDA), and RADAR. However, since these sensors have large size, therefore they cannot be incorporated into UAVs.

Researchers in [26] have studied the latest trends in reactive inertial sensors. Results obtained proved huge time delays and slow response while using single sensors for flight control during gusty airflows and necessitated the use of multisensor systems for attitude control. Another research [27] showed that conventional reactive attitude sensors have very slow response times for attitude control during turbulent environments. They presented a solution to the above-mentioned delays and developed novel bioinspired sensors, which provide phase advanced information of disturbances thereby improving response time of actuators.

Synthetic jet is another method for gust alleviation. These jets when combined into the wing extend the flight envelope of the aircraft to higher angles of attack through active flow control and therefore reduce flight instability [28]. By producing these jets, the boundary layer remains attached in gusty wind conditions thereby enabling the UAV to maintain stability. However, since the size of SJAs is large enough to be incorporated in UAVs, their exploration for small scale aircrafts remains an open field for researchers. Similarly, vortex generators that produce microvortices on the surface of wing in order to avoid boundary layer separation are also the variant of active GMS techniques for larger aircrafts [29]. However, due to size constraints, their applicability for UAVs remains a major question.

Yeo et al. [30] have proposed flow aware wing design combining aerodynamic, computational, electrical, and mechanical elements to have improved flight during turbulent flows. Each wing is upgraded with an independent processor in order to actuate a dedicated control surface. Numerical simulation results have verified that the flow aware design of wings show a $22 \%$ decrease in roll angle deviancies and $18 \%$ drop in roll rate deviations in presence of turbulence.

Blower and Wickenheiser in [20] have presented primary- and secondary-feathers-inspired biomimetic flow sensors for fixed wing UAVs and discovered their suitability for usage as an active GMS. The design showed significant improvement in stability characteristics of UAVs; however, their usage for FUAVs has not been discussed and leaves an open gap for researchers.

The detailed study of birds has revealed an interesting fact that during high turbulent airflows and gusty winds, birds take on an intermittent flight, i.e., nonflapping phase. The covert feathers during these intermittent gliding flights get activated to alleviate gusts as shown in Figure 2 [31].

Inspired by the biological covert feathers of birds, this research presents a novel distributed GMS for FUAV. GMS comprises EM covert feathers integrated in flapping wings of UAV. GMS activates only at the time of turbulent airflows to mitigate gust, while at all other instants it remains tightly attached with wing to retain airfoil overall profile. It provides various flight advantages, including better maneuverability and enhanced stability during adverse wind environments.

Preliminary version of this research is presented by authors in $[32,33]$ in which only the development of GMS and its effectiveness is studied by incorporating it in a rigid wing and performing simulations. The efficacy of the proposed design by modeling the complete FUAV having GMS installed in it needed to be ascertained. Therefore, the substantial enhancement of current research is as follows. First, we present comprehensive model of a complete FUAV comprising the main body and its allied accessories. These accessories include flapping mechanism, wings, and the propulsion system which comprises battery, motor, and the gear box. Second, GMS is incorporated in the model of FUAV and a complete multibody model of gust-mitigating FUAV is presented. We utilize bond graph modeling (BGM) for developing the complete model and for performing simulations of gust-mitigating FUAV. 20-SIM software is used in this research for modeling. Furthermore, we generate state space equations for in-depth analysis of internal dynamics. Lastly, we simulate multibody model to check its 


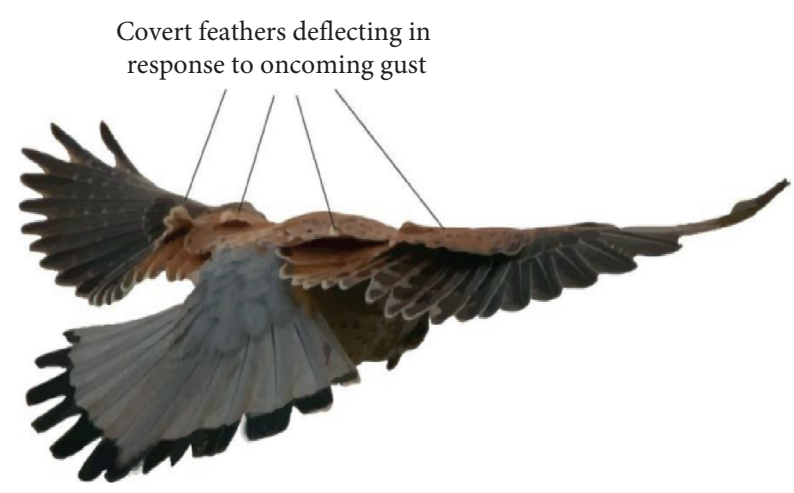

Figure 2: Deflection of covert feathers during turbulence [31].

accuracy and efficacy and also compare results with published experimental researches for validation of proposed scheme.

The remaining paper is organized as follows. In Section 2, the design of gust-mitigating FUAV is presented. Section 3 covers the creation of bond graph model (BGM) of FUAV subsystems leading to the formulation of a multibody model of complete gust-mitigating FUAV. To validate the accuracy of proposed design and to check its correctness, comparison of results with experimental studies and subsequent discussions are carried out in Section 4. The final section includes conclusions and future work.

\section{FUAV Design}

The prototype FUAV under study is Festo's Smart Bird [34]. The Festo bird is having $2.2 \mathrm{~m}$ wing span and $0.28 \mathrm{~m}$ chord length. Dynamic model of the system under investigation can be developed considering that the FUAV is composed of subsystems namely the main body, motors, the flapping mechanism, rigid wings, and GMS.

The flapping system comprises main structure, rigid beam wings, two DC motors driven by two batteries, and pair of crank rod mechanisms. A sketch of this flapping mechanism is illustrated in Figure 3. The flapping system also consists of an arm added to slider bar as well as to wings to generate the motion perpendicular to wing surface. The top angular velocity of wing is about $12 \mathrm{rad} / \mathrm{s}$.

The proposed GMS consists of 16 biomimetic EM covert feathers. Eight are incorporated in the wing's top surface and eight on the wing's bottom surface. Single EM covert feather comprises flap, hinge, mechanical linkage, spring, piezoelectric transducer (PZT), controller, and the voice coil actuator. The piezoelectric transducers having small size and multiple functionalities are utilized in EM feathers due to the size limits of wings. Moreover, PZTs can have dual functionality at the same time, i.e., acting as sensors as well as actuators.

The FUAV wing is composed of a skeletal structure equipped with ribs and spars to carry loads. The design of GMS ensures the wings to retain the airfoil's overall shape throughout flapping phases as EM covert feathers remain firmly attached to wings. At the time of high turbulence, FUAV resorts to intermittent flight and GMS activates resultantly the EM feathers rotating to allow strong gusts to transpire through the airframe with little impedance.

Figure 4 illustrates EM covert feather's internal working. The flap rotates as a response to incident gust and gives signal to PZT that is acting as a sensor through mechanical linkage and spring. After receiving gust signal, the PZT now acts as an actuator and produces an output signal equivalent to gust experienced and gives it to controller which in turn generates desired control output. This control output, i.e., current, is forwarded to voice coil actuator. Voice coil actuator moves out the shaft inside it and applies force on the flap that deflects out of the wing. Consequently, the gust flows through the EM covert feathers with very minimal interaction with the wing's cross-sectional area.

Every EM covert feather is having a closed loop feedback design. The GMS has a separate control and is not dependent on the main UAV controller. This design allows local data analysis and control, thereby decreasing response times compared to traditional present day gust alleviation designs that have delayed reaction times. During larger turbulence, multiple feathers actuate since single feather response is not enough. This minimizes stress on a single EM feather, since the incoming gust is spread over a certain region of the wing instead of concentration on a single point.

\section{Bond Graph Formulation and Derivation of Dynamic Equations}

Modeling is the process of interpreting scientific problems from an application field into tractable mathematical formulations including its construction and working. This formulation of model helps in developing scientific understanding which assists in testing the effects of changes in a system and offers insight, solutions, and direction beneficial for the original application. A mathematical model generally defines a system by a set of equations and variables that form relationships between the variables. Modeling renders solutions by providing clear understandings into complex systems. [36].

Bond graph models are a domain independent graphical depiction of dynamic behavior of physical systems. Meaning by the systems from various domains, i.e., electrical, mechanical, hydraulic, thermodynamic, acoustical, etc., is labeled in the same way. The basic concept is that bond graphs work on energy exchange between various domains. Similarities between domains are nothing more than just mathematical equations being analogous, i.e., the utilized physical concepts are the same. Bond graph modeling is a potent tool for modeling engineering systems, particularly when diverse physical domains are present. Additionally, bond graph submodels can be reused smartly, since bond graph models are noncausal. If the submodels are seen as objects, we can easily say that the bond graph modeling is a type of object-oriented modeling of physical systems [37].

3.1. Main Body BGM. The FUAV's main body is taken as 6 DOF rigid body which can perform both rotational and translational motion. The analysis of motion of rigid body in 


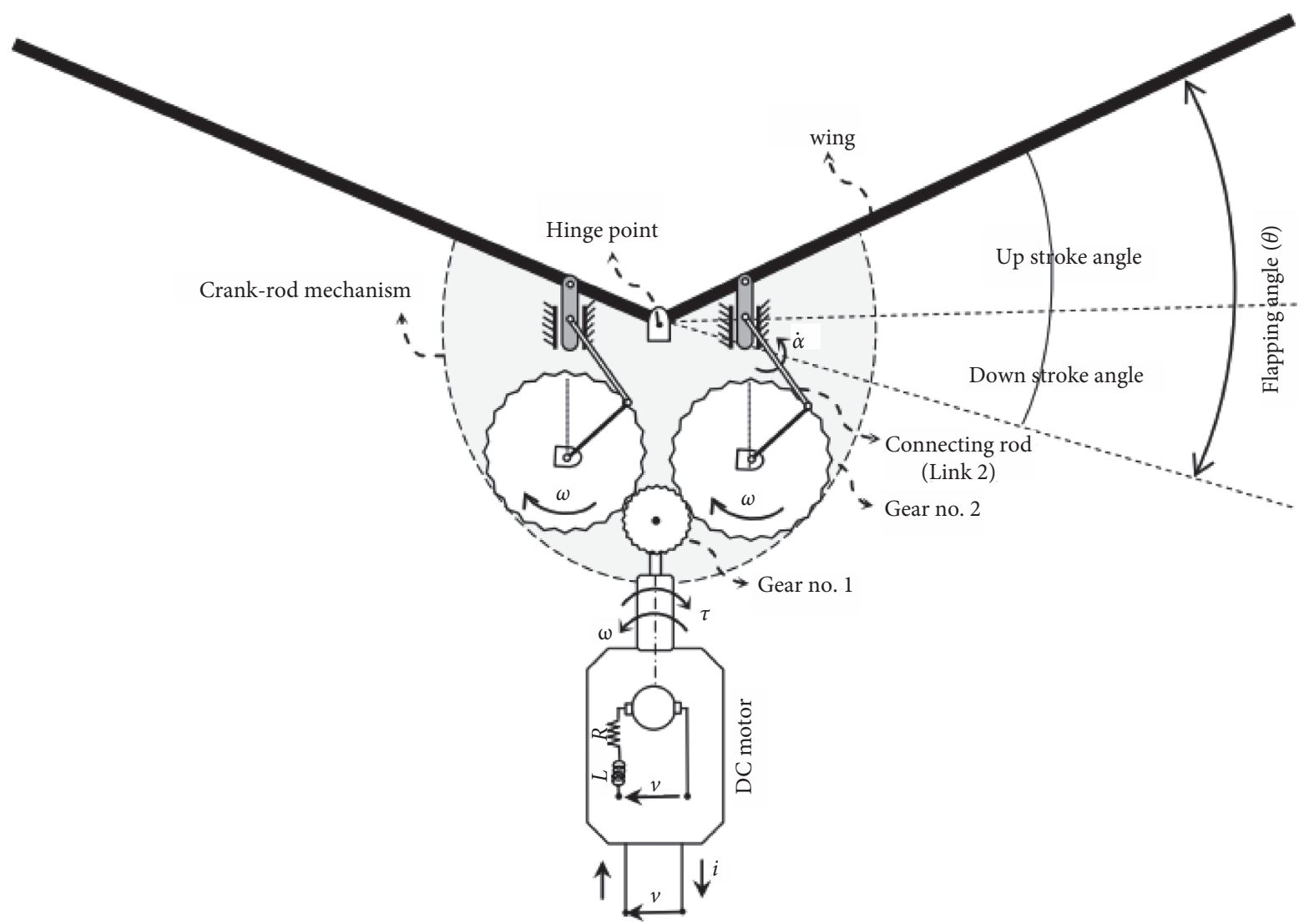

FIGURE 3: Sketch of present flapping mechanism [35].

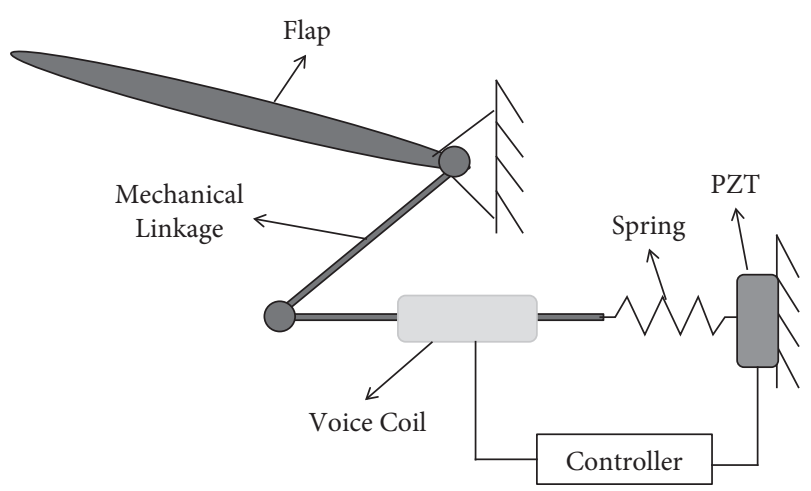

FIGURE 4: Electromechanical covert feather.

space produces equations given below based on Newton's second law of motion [37]:

$$
\begin{gathered}
\mathbf{F}=\frac{\partial \mathbf{p}}{\partial t}+\omega \times p, \\
\boldsymbol{\tau}=\frac{\partial \mathbf{p} J}{\partial t}+\omega \times p J,
\end{gathered}
$$

where $p$ is linear momentum, $p_{j}$ is angular momentum, $F$ is the force acting on the body, $\tau$ is the torque, and $\omega$ is the angular velocity of the main body. These two equations, i.e., (1) and (2), derived from Newton's second law of motion, help attain the Euler's equation given in (3)-(8). The analytical solution of these Euler's equations can be found in certain cases; however, their general solution cannot be found [37]. Finally, using these six equations, the BGM of the main body is formed.

$$
\begin{aligned}
& \dot{p}_{x}=F_{x}+m \omega_{z} \frac{P_{y}}{m}-m \omega_{y} \frac{P_{z}}{m}, \\
& \dot{p_{y}}=F_{y}+m \omega_{x} \frac{P_{z}}{m}-m \omega_{z} \frac{P_{x}}{m}, \\
& \dot{p}_{z}=F_{z}+m \omega_{y} \frac{P_{x}}{m}-m \omega_{x} \frac{P_{y}}{m}, \\
& \dot{p J_{x}}=\tau_{x}+J_{y} \omega_{y} \frac{P J_{z}}{J_{z}}-J_{z} \omega_{z} \frac{P J_{y}}{J_{y}}, \\
& \dot{p J_{y}}=\tau_{y}+J_{z} \omega_{z} \frac{P J_{x}}{J_{x}}-J_{x} \omega_{x} \frac{P J_{z}}{J_{z}}, \\
& \dot{p J_{z}}=\tau_{z}+J_{x} \omega_{x} \frac{P J_{y}}{J_{y}}-J_{y} \omega_{y} \frac{P J_{x}}{J_{x}} .
\end{aligned}
$$

Figure 5 shows the final BGM of the main body with a general motion in 3 dimensional space. Six state space equations are obtained from the above-mentioned BGM because the number of energy storing elements is 6 . The state 


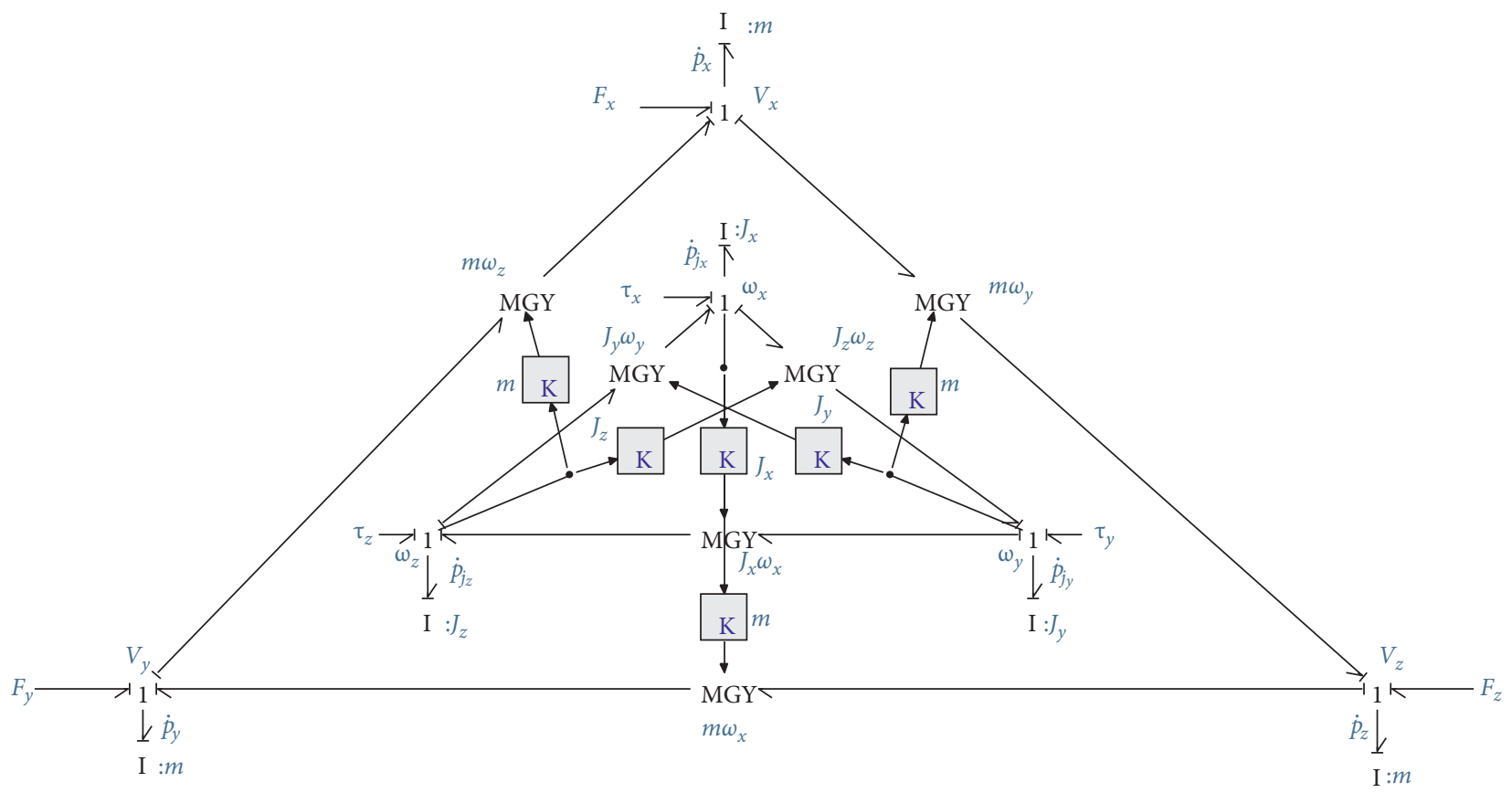

Figure 5: BGM of the main body of FUAV.

variables comprise generalized momentum $p_{x}, p_{y}, p_{z}, p_{j x}, p_{j y}$, and $p_{j z}$ at every inertia element.

3.2. DC Motors BGM. The DC motors are powered by a battery source and are used to change electrical energy into mechanical energy. It comprises an electromechanical coupling and the armature which further consists of inductance and resistance elements. The back EMF of the motors is presented as a gyrator in the BGM [37]. The BGM of the DC motor is developed using the above-mentioned description and is presented Figure 6.

3.3. Flapping Mechanism BGM. The flapping motion of the FUAV under study is attained through slider crank mechanism. This mechanism comprises two rods linked together and an arm which is hinged at $90^{\circ}$ angles to the rotating shaft. The reciprocating movement is transmitted to and received from this shaft and is used for conversion of rotational motion into reciprocating motion and also vice versa. Input to the crank rod, i.e., velocity, is applied as a source of flow. The corresponding BGM is shown in Figure 7. 1-junction $(1 \dot{J})$ is used for depicting the motion of the crank. The inertia of the crank about its axis is shown as $I$ element and the linear velocity of the connecting rod as $(1 \dot{x}, 1 \dot{y})$, whereas $I$ elements show the mass of connecting rod. 1 -junction $(1 \dot{\alpha})$ and modulated transformers (MTF) are used for rotational motion of the link [37]. Furthermore, two inertial elements representing mass and mass moment of inertia of the components are dovetailed to the corresponding junctions.

3.4. Rigid Beam Wing BGM. Dynamics of the wings are modeled as a rigid beam in transverse motion that is pivoted at one end. In this case, the wing's vertical displacement at the end point is calculated by $[37,38]$

$$
y=l \sin \theta
$$

where $y$ is the displacement, $\theta$ is the flapping angle, and $l$ is the wing span respectively. The effort and flow relation can be described as follows [39]:

$$
\begin{aligned}
V_{y} & =(l \cos \theta) \omega, \\
\left(x_{1} \cos \theta\right) F & =\tau,
\end{aligned}
$$

where $V_{y}$ is the vertical direction velocity, $F$ is the force, and $\tau$ is the torque, respectively. Figure 8 demonstrates the BGM of wing under vertical force, which is gust $(S f) . l \cos \theta$ and $x_{1} \cos \theta$ are the transformer modulus relating translational quantities to rotational quantities.

3.5. BGM of Gust Mitigation System (GMS). In this section BGM of a GMS is presented. Detailed development of BGM of GMS starting from single electromechanical (EM) covert feather will not be discussed as the same has been extensively investigated in previous work [32, 33].

BGM of EM covert feather is shown in Figure 9. The overall order of the model is eight since there are eight energy storing elements. There is one disturbance input, i.e., $S_{f}$ (source of flow) which depicts gust incident on the feather. And there are two controllable inputs, i.e., MSf (modulated source of flow) and MSe (modulated source of effort). These inputs form part of input vector $\vec{u}(t) . \mathrm{MS}_{e}$ is actually the force applied on the linkage, whereas MSf is the current being input to the voice coil actuator.

The BGM obtained is used to formulate state space equations given as (11)-(19). The state variables comprise generalized momentum $p_{1}, p_{2}, p_{3}$ at every inertia element 


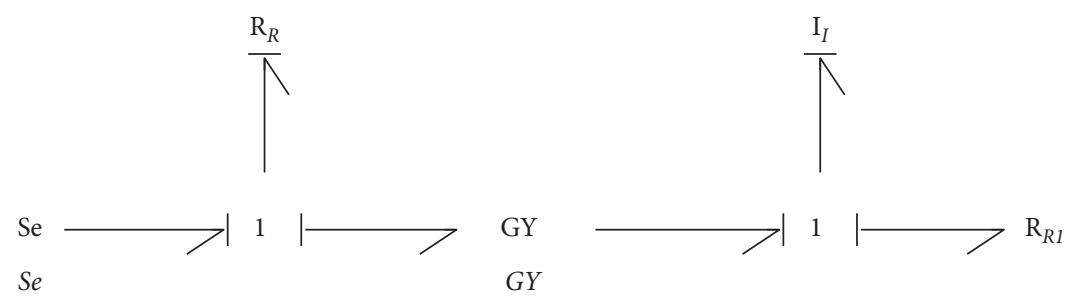

FIGURE 6: BGM of a DC motor.

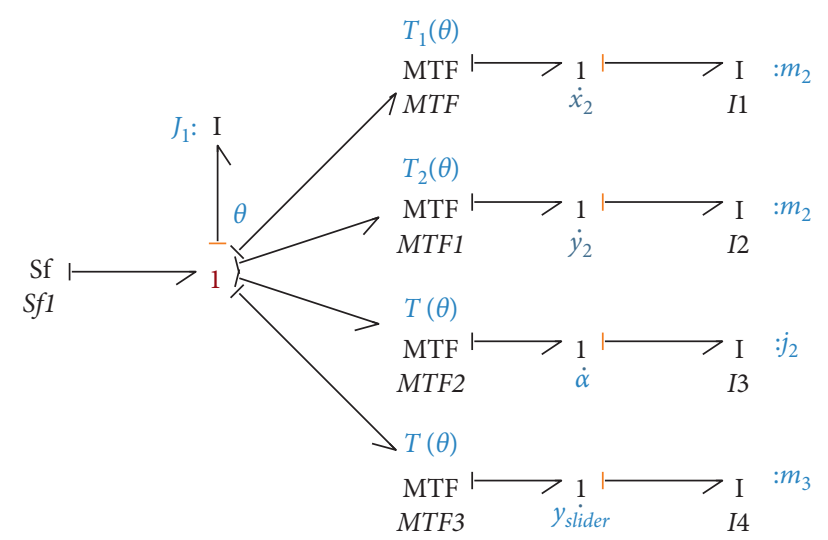

FIgURE 7: BGM of a flapping mechanism.

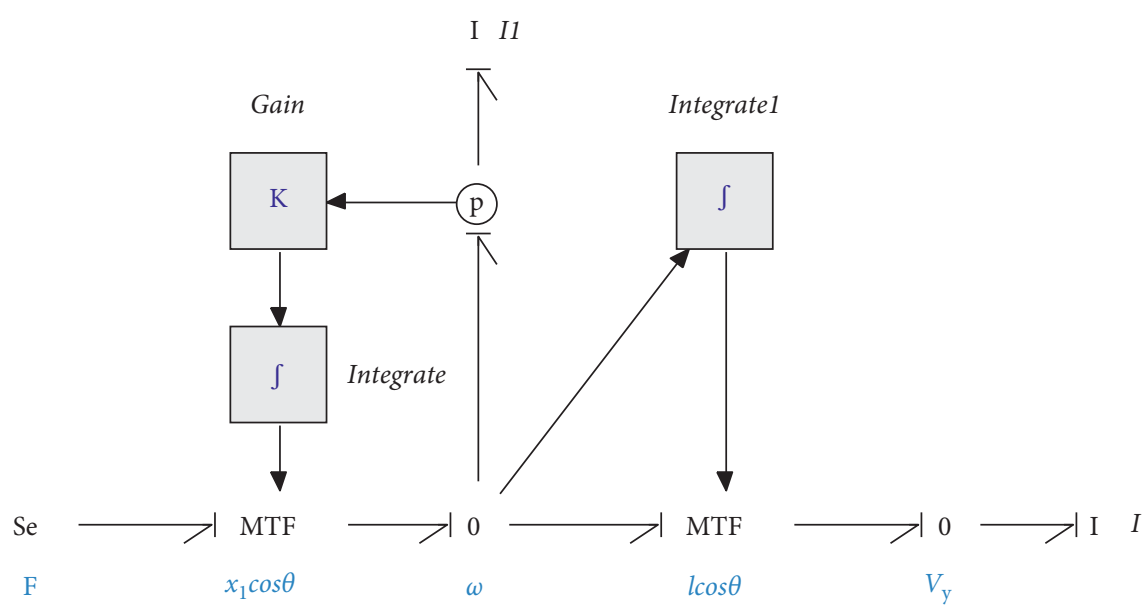

FIgURE 8: BGM of a wing.

and generalized displacement $q_{1}, q_{2}, q_{3}, q_{4}$ at every compliance element. State variable $q_{5}$ is the state of displacement sensor used in the bond graph.

$$
\begin{aligned}
& \dot{p_{1}}=i c \cdot p_{3}+i c \cdot q_{3}, \\
& \dot{q}_{1}=\frac{1}{I_{1}} \cdot p_{2}, \\
& \dot{p_{2}}=\frac{i c}{l} p_{3}+\frac{i c}{l} q_{3}-\frac{1}{C} q_{1}-\frac{1}{C_{1}} q_{2}-\frac{m}{C_{2}} q_{4},
\end{aligned}
$$

$$
\begin{aligned}
& \dot{q}_{2}=\frac{1}{I_{1}} \cdot p_{2}, \\
& \dot{p}_{3}=q_{5}, \\
& \dot{q}_{3}=S_{f}-\frac{1}{l \cdot I_{1}} p_{2}-\frac{1}{I} p_{1}, \\
& \dot{q}_{4}=\frac{m}{I_{1}} p_{2}-\frac{R}{C_{2}} q_{4},
\end{aligned}
$$




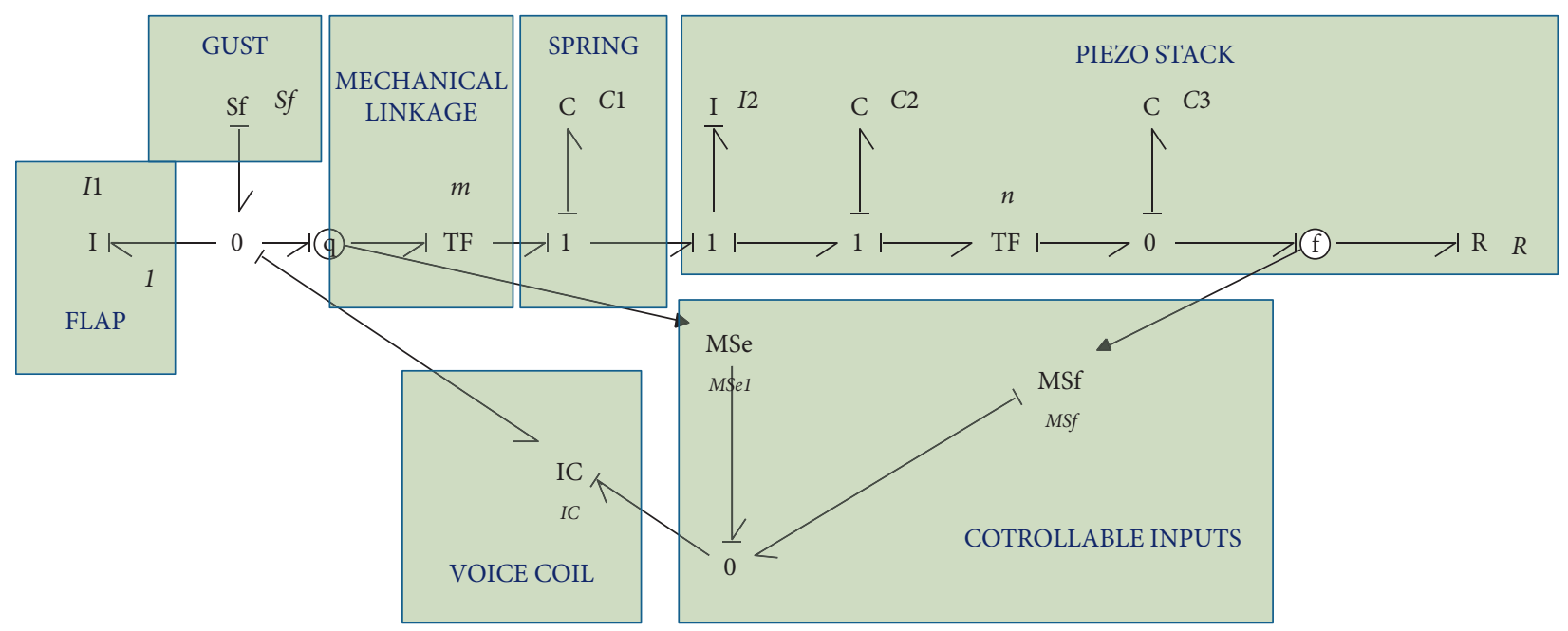

Figure 9: BGM of EM feather.

$$
\dot{q}_{5}=\frac{1}{l \cdot I_{1}} p_{2}
$$

Figure 10 shows the BGM of GMS comprising 16 EM covert feathers.

3.6. Complete BGM of a Gust-Mitigating FUAV. To study the efficacy and correctness of the proposed GMS after incorporation in both wings of FUAV, the complete BGM of the FUAV comprising main body and GMS incorporated rigid wings driven by two DC motors and slider crank flapping mechanism is taken into account. The complete BGM of the Gust-Mitigating FUAV is developed by joining the BGM of the subsystems presented in previous sections using appropriate junctions and is illustrated in Figure 11. It is important to mention here that complete BGM of gust-mitigating FUAV has been reduced to $8 \mathrm{EM}$ covert feathers per wing to avoid modeling complexity, since complete FUAV model comprising 16 feathers per wing results in $260^{\text {th }}$ order model and is challenging to simulate. Moreover, this reduced model presents a baseline and is sufficient to prove efficacy of the proposed GMS design. Further research can be carried out to find out optimal numbers of feathers that can be added in a wing.

The BGM in Figure 11 helps us compute state space of gust-mitigating FUAV. The state matrix $\vec{x}(t)$ contains generalized momentum of inertia elements and generalized displacement of compliance elements. There are 17 disturbance inputs depicting gusts which are applied to each EM covert feather. Additionally, gust is applied to rigid wing as well as a source of flow, i.e., $S_{f}$ at 0 junction and this also forms part of the disturbance input vector. There are 34 controllable inputs including 2 sources of efforts $\left(S_{e}\right)$ represent the DC motors, 16 are displayed as modulated flow source, i.e., $M S_{f}$, and 16 are presented as modulated effort source, i.e., $M S_{e}$. These 34 controllable inputs form part of the input vector $\vec{u}(t)$. The final state matrix A comes out to be of $132 \times 132$ order, input gain matrix B comes out to be of $132 \times 34$ order, and the output gain matrix $\mathrm{C}$ comes out to be of $34 \times 132$ order.
3.7. Nonlinear Dynamic Modeling. In this research, the scope remains only to simulate vertical gust which produces the lift force in the FUAV. This lift force is integrated in BGM as flow source $\left(S_{f}\right)$. The aerodynamic lift force being incident on the wings of FUAV produces upward movement, i.e., $z$ direction. The modulated transformer, i.e., MTF, is utilized in the BGM as shown in Figure 11 for conversion of wing motion into FUAV's displacement in $z$ direction.

The comprehensive modeling of Gust-mitigating FUAV by integrating all aerodynamic and structural elements is complicated. For simplification, certain assumptions are made that include neglecting a wide range of aerodynamic forces encountered by FUAV being explored in the latest studies, i.e., thrust, drag, wing's wake, rotational inertia, circular rotation, rotational lift, leading edge vortex, viscous friction, and added mass. Also, we model flapping wing as a rigid beam, and further insight into the wing's flexibility remains out of scope of the present work. Moreover, nonlinearity due to inherent system imperfections giving rise to hidden dynamics and the associated control strategy to optimally utilize these nonlinearities for positive impact on the overall system as presented by [40] remains open for research. Addition of several boundary conditions, various input forces and moments, extra degrees of freedoms and moreover incorporation of flexibility, and altering the wing's vibrational modes need to be further explored. Also, oscillations rise in the FUAV by means of unmodeled nonlinearities, and delays leading to dominant scenarios of bifurcations and chaos as studied in [35] are out of the scope of the present research.

\section{Results Validation and Discussion}

In order to ascertain the correctness of BGM of gustmitigating FUAV developed in the above section, we use three vertical gust speeds $(35 \mathrm{~m} / \mathrm{s}, 25 \mathrm{~m} / \mathrm{s}, 15 \mathrm{~m} / \mathrm{s})$ on three feathers (feather no 1 , feather no 2 , and feather no 3 ) installed on the right wing of FUAV. Figure 12 depicts the voice coil actuator current of these 3 EM feathers. The peak values currently depicted in the figure are in correct range 


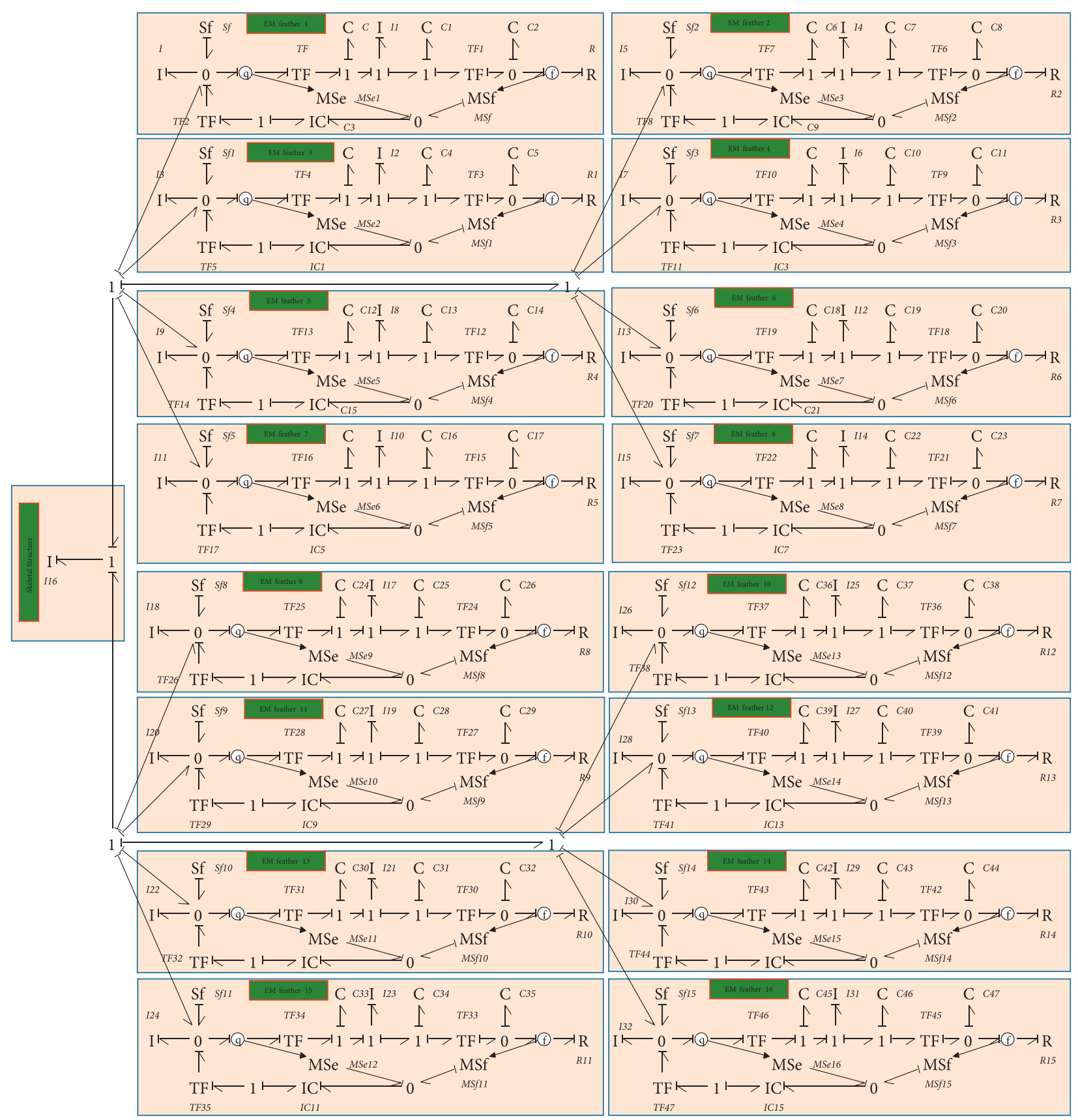

Figure 10: BGM of 16 EM feathers GMS.

and are directly proportional to the gust force being applied on feathers. Figure 13 depicts the flapping angle of FUAV given in radians.

In order to prove efficacy of the proposed design, two simulation scenarios are considered. First the FUAV without GMS is simulated by applying $25 \mathrm{~m} / \mathrm{s}$ gust on its wing, and movement in $z$ direction is observed. Second, the same gust is applied on the FUAV with GMS installed in it and the corresponding motion in $z$ direction is analyzed. The displacement in $z$ direction of both the simulation scenarios is illustrated in Figure 14. The corresponding roll angles of FUAVs in both scenarios are also shown in Figure 15.
It can be clearly seen that the gust-mitigating FUAV has successfully alleviated the gust to $32 \%$ because of the actuation of EM covert feathers installed on the wing as expected. The GMS-installed FUAV has displaced only $11.2 \mathrm{~m}$ in comparison to $16.5 \mathrm{~m}$ displacement for the model without GMS. Likewise, the roll angle of FUAV with GMS has reduced to $0.21 \mathrm{rad}$ in comparison to $0.31 \mathrm{rad}$ for the model without GMS. The above-mentioned results confirm the anticipated utility of the proposed gust-mitigating FUAV design. Moreover, it is pertinent to mention here that, in the preliminary version of this research by authors in $[32,33]$, the installation of GMS in the rigid wing helped mitigate the gust up to $50 \%$ and here in this research the mitigation in 


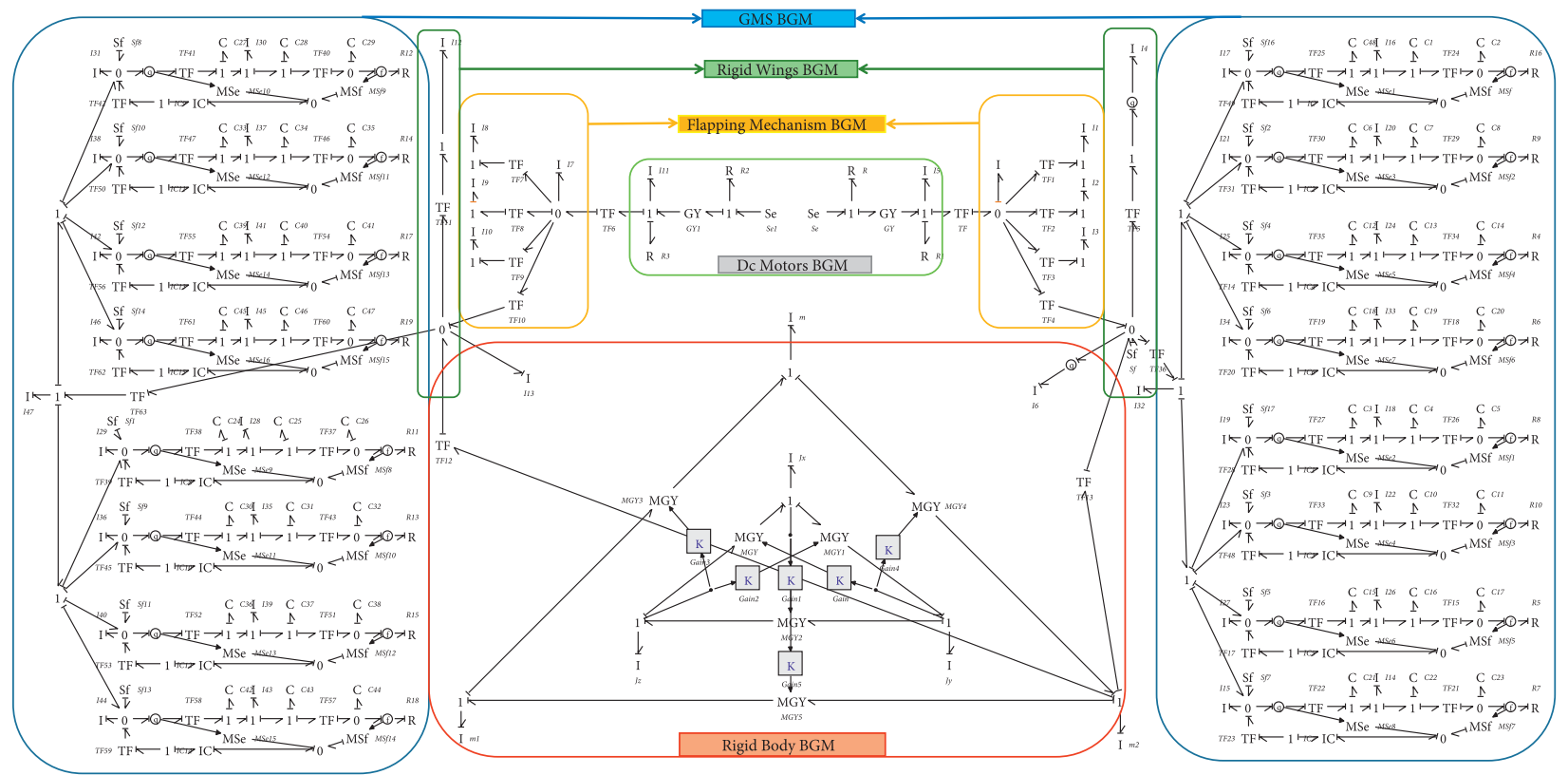

Figure 11: BGM of a complete gust-mitigating FUAV.

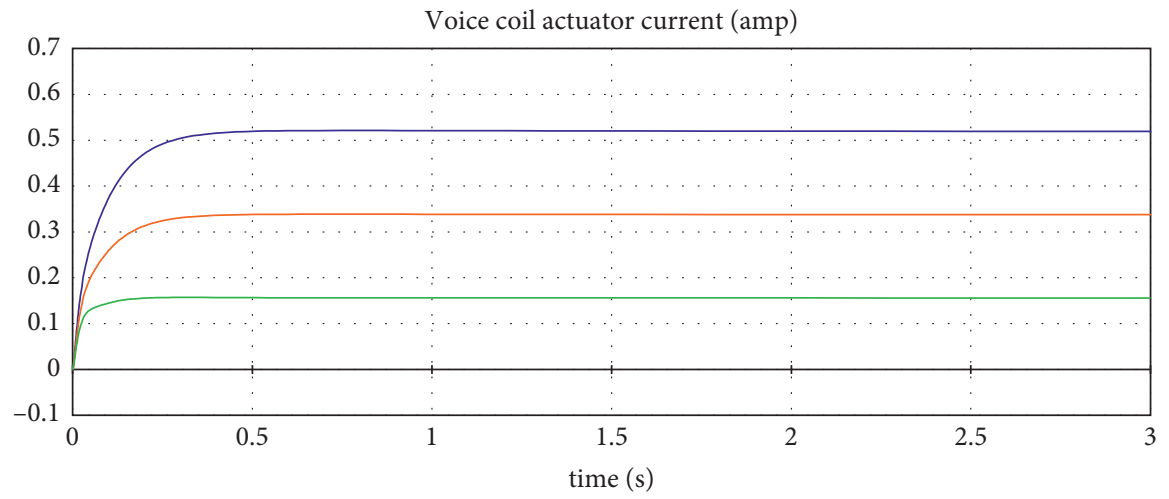

- Feather 1 at gust speed $35 \mathrm{~m} / \mathrm{s}$

— Feather 2 at gust speed $25 \mathrm{~m} / \mathrm{s}$

_ Feather 3 at gust speed $15 \mathrm{~m} / \mathrm{s}$

Figure 12: Voice coil actuator current.

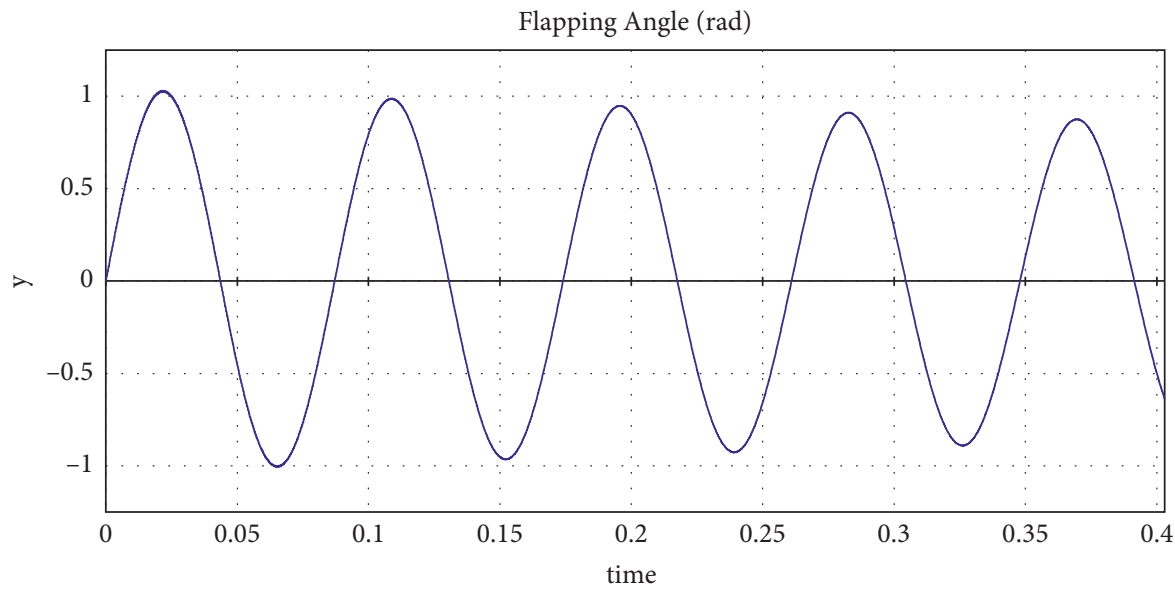

FIGURE 13: Flapping angle of FUAV. 
Vertical Displacement (m)

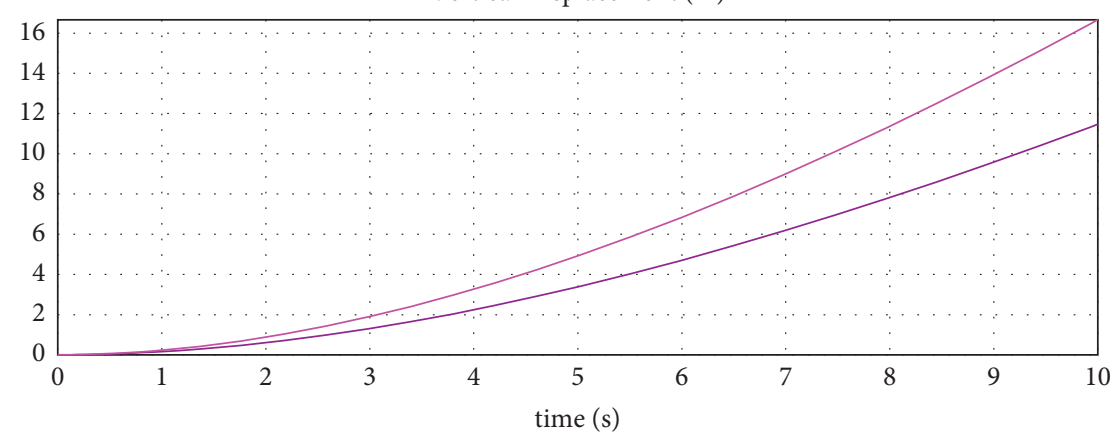

- With GMS

- Without GMS

FIgURE 14: Displacement of FUAV in vertical direction.

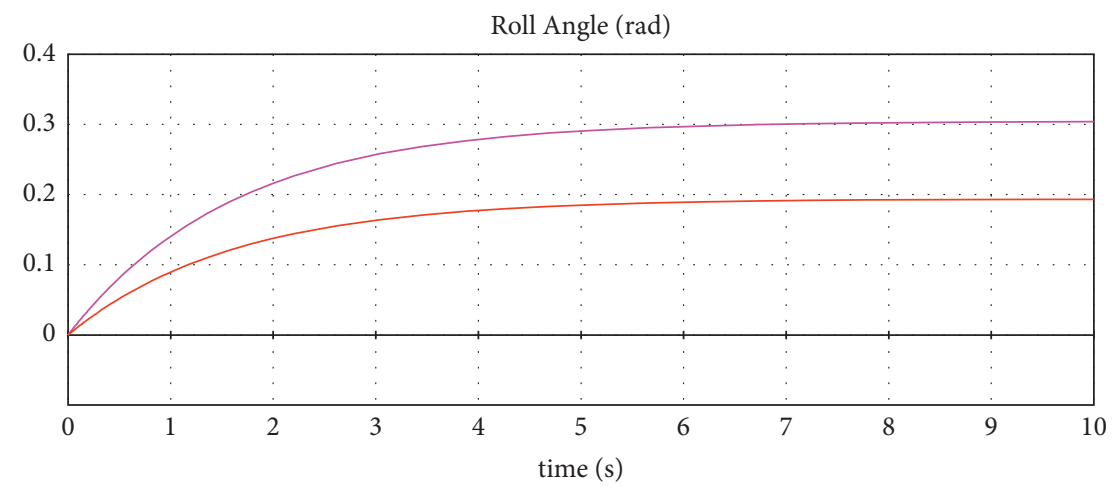

- Without GMS

- With GMS

Figure 15: Roll angle of FUAV (with and without GMS).

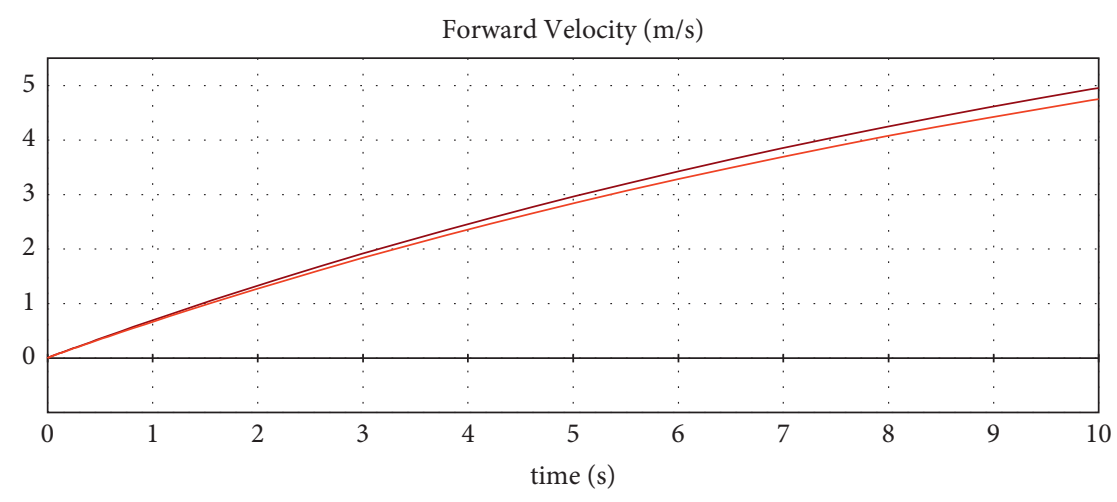

- Present

- Experimental

FIGURE 16: Comparison of forward velocities in present work and experimental research [41].

complete FUAV is $32 \%$ which is exactly within the anticipated range.

Figure 16 shows the comparison of forward velocities of FUAV in present research and study by [41]. Furthermore, results attained in current study are compared to the findings of experimental research by [41] and are summarized in Table 1. Very close agreement among the results of FUAV with GMS and FUAV without GMS, acquired in current work and experimental findings, endorses accuracy and validity of the proposed design.

For further insight into model internal dynamics, gust speed on the right wing $\left(S_{f}\right)$ is used as input and force 
TABLE 1: Comparison between present work and experimental research.

\begin{tabular}{lccc}
\hline & & $Z$ displacement (m) \\
\hline \multirow{2}{*}{ Current work } & Without GMS & 16.5 \\
& With GMS & 11.2 & 16.9 \\
\hline
\end{tabular}

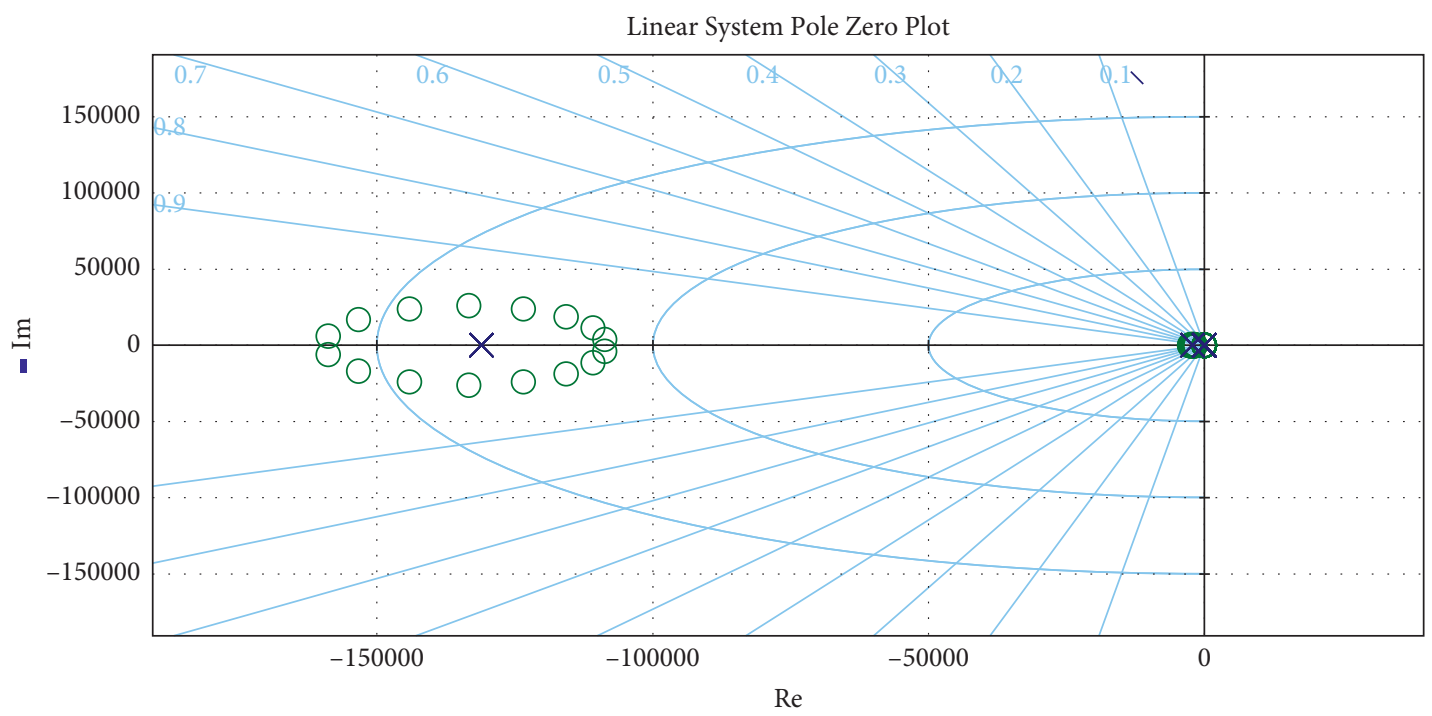

Figure 17: Pole-zero (PZ) plot.

TABle 2: Parameters of model.

\begin{tabular}{|c|c|c|}
\hline Component & Description & Values \\
\hline \multicolumn{3}{|l|}{ Motors } \\
\hline Voltage source & Electrical & $7.2 \mathrm{~V}$ \\
\hline Armature resistance of the motors & Electrical & $5.1 \Omega$ \\
\hline Gyrator ratio of motors & Electrical & 0.00813 \\
\hline Damping of motors & Mechanical & $0.00068 \mathrm{~N}-\mathrm{s} / \mathrm{m}$ \\
\hline Mass of motors & Mechanical & $0.021 \mathrm{~kg}$ \\
\hline \multicolumn{3}{|l|}{ Gears } \\
\hline Ratio of gears & Mechanical & 0.112 \\
\hline \multicolumn{3}{|l|}{ Flapping mechanism } \\
\hline Mass moment of inertia of crank & Mechanical & $0.009 \mathrm{~kg} / \mathrm{m}^{2}$ \\
\hline Transformer ratio of connecting rod & Mechanical & 2 \\
\hline Transformer ratio of linkages & Mechanical & 1 \\
\hline Mass of connecting rod & Mechanical & $0.03 \mathrm{~kg}$ \\
\hline Mass moment of inertia of connecting rod & Mechanical & $0.006 \mathrm{~kg} / \mathrm{m}^{2}$ \\
\hline \multicolumn{3}{|l|}{ Rigid beam wing } \\
\hline Mass of rigid beam & Mechanical & $0.4 \mathrm{~kg}$ \\
\hline Mass moment of inertia of rigid beam & Mechanical & $0.024 \mathrm{~kg} / \mathrm{m}^{2}$ \\
\hline Transformer ratio of rigid beam & Mechanical & 1 \\
\hline \multicolumn{3}{|l|}{ Main body } \\
\hline Mass of body & Mechanical & $0.15 \mathrm{~kg}$ \\
\hline Mass moment of inertia $\left(J_{x}, J_{y}, J_{z}\right)$ & Mechanical & $0.002,0.004,0.003 \mathrm{~kg} / \mathrm{m}^{2}$ \\
\hline Gust speed & Mechanical & $25 \mathrm{~m} / \mathrm{s}$ \\
\hline \multicolumn{3}{|l|}{ GMS } \\
\hline \multicolumn{3}{|l|}{ Flap } \\
\hline Mass of flap & Mechanical & $0.018 \mathrm{~kg}$ \\
\hline Mass of skeletal structure & Mechanical & $0.098 \mathrm{~kg}$ \\
\hline Gust velocity on feather & Mechanical & $25 \mathrm{~m} / \mathrm{s}$ \\
\hline Voice coil actuator & & \\
\hline
\end{tabular}


TABLE 2: Continued.

\begin{tabular}{lcc}
\hline Component & Description & Values \\
\hline Inductance & Electrical & $0.89 \mathrm{H}$ \\
Stiffness & Mechanical & $0.589 \mathrm{kN} / \mathrm{m}$ \\
Piezoelectric stack & & $5 \Omega$ \\
Resistance between amplifier and PZT & Electrical & $0.008 \mathrm{~kg}$ \\
Mass of stack & Mechanical & $0.024 \mathrm{kN} / \mathrm{m}$ \\
PZT spring stiffness & Mechanical & $1.5 \times 10^{-7} \mathrm{~F}$ \\
PZT equivalent capacitance & Electrical & 0.478 \\
Coupling ratio & Electrical & $0.03 \mathrm{kN} / \mathrm{m}$ \\
Spring & & \\
Spring stiffness & Mechanical & 0.2 \\
Mechanical linkage & & \\
Transformer ratio & Mechanical & \\
\end{tabular}

acting on wing $\left(\mathrm{I}_{6}\right)$ is used as output. Linearizing the model for this input and output in 20-SIM software generates $132^{\text {nd }}$ order model. Figure 17 illustrates pole-zero plot of the model and displays that multiple poles are at origin and some of the poles are in the right half plane and thus the system is unstable. Moreover, step response indicates diverging response further endorsing the internal unstable dynamics of the system under study, whereas in the system in which GMS was installed in rigid wing in the preliminary version of this research in $[32,33]$, the internal dynamics were stable.

The values of elements of BGM of gust-mitigating FUAV presented in Figure 11 are shown in Table 2. It must be noted that elements of all the 16 EM feathers are the same as one EM feather and are described in detail in previous work [33].

\section{Conclusions}

We propose a design of a new Gust Mitigation System (GMS) for flapping wing UAV (FUAV) inspired from covert feathers of birds. We develop a complete Bond Graph Model (BGM) of a FUAV containing the main rigid body, flapping system, GMS-installed wings, and the power system comprising of the battery, motor, and a gear combination. Addition of electromechanical (EM) covert feathers on the top and bottom wing surfaces of FUAV decreases the gusting forces being exerted on FUAV body and thereby offers a novel strategy of active gust mitigation for FUAVs.

Simulations performed reveal hierarchal response generation capability of feathers and also the unstable internal dynamics. The results further show successful mitigation of gust to $32 \%$ and therefore validate the effectiveness of the proposed model. Moreover, the strong agreement between experimental results and present results validates the accuracy of the proposed design and developed model.

Further extension to the present study is planned including study of various control schemes for stabilizing the unstable internal dynamics of the gust-mitigating FUAV. Finally, designing of the FUAV based on the validated proposed BGM model will be done as an effort to contribute to research on active gust mitigation systems for FUAVs.
Abbreviations
GMS: Gust mitigation system
BGM: Bond graph model
FUAV: Flapping wing UAV
GAS: Gust alleviation system
PZT: Piezoelectric transducer
EM: Electromechanical
UAV: Unmanned aerial vehicle
UAS: Unmanned aircraft system
CFD: Computational fluid dynamics
Sf: $\quad$ Source of flow
Se: $\quad$ Source of effort
MSf: Modulated source of flow
MSe: $\quad$ Modulated source of effort
TF: $\quad$ Transformer
GY: Gyrator
SJA: $\quad$ Synthetic jet actuators.

\section{Data Availability}

The data that support the findings of this study are available from the corresponding author upon reasonable request.

\section{Conflicts of Interest}

The authors have confirmed no potential conflicts of interest with respect to the research, authorship, and/or publication of this article.

\section{References}

[1] S. Watkins, M. Thompson, B. Loxton, and M. Abdulrahim, "On low altitude flight through the atmospheric boundary layer," International Journal of Micro Air Vehicles, vol. 2, no. 2, pp. 55-68, 2010.

[2] S. Watkins, J. Milbank, B. J. Loxton, and W. H. Melbourne, "Atmospheric winds and their implications for microair vehicles," AIAA Journal, vol. 44, no. 11, pp. 2591-2600, 2006.

[3] R. H. Shaw and U. Schumann, "Large-eddy simulation of turbulent flow above and within a forest," Boundary-Layer Meteorology, vol. 61, 1992. 
[4] M. P. Coutts and J. Grace, Wind and Trees, Cambridge University Press, Cambridge, UK, 1995.

[5] G. Katul, C. I. Hsieh, G. Kuhn, and D. Ellsworth, "Turbulent eddy motion at the forest-atmosphere interface," Journal of Geophysical Research, vol. 102, pp. 409-413, 1997.

[6] C. J. Blower and A. M. Wickenheiser, "Biomimetic feather structures for localized flow control and gust alleviation on aircraft wings," in Proceedings of the 21st International Conference on Adaptive Structures and Technologies, PA, USA, October 2010.

[7] J. S. Mccarley and C. D. Wickens, Human Factors Concerns in UAV Flight, University of Illinois at Urbana-Champaign Institute of Aviation, Aviation HumanFactors Division, Champaign, IL, USA, 2004.

[8] C. Galiński and R. Żbikowski, "Some problems of micro air vehicles development," Bulletin of the Polish Academy of Sciences; Technical Sciences, vol. 55, pp. 91-98, 2007.

[9] C. R. Galinski, "Gust resistant fixed wing micro air vehicle," Journal of Aircraft, vol. 43, pp. 1586-1588, 2006.

[10] P. Lissaman, "Effects of turbulence on bank upsets of small flight vehicles," in Proceedings of the 47th AIAA Aerospace Sciences Meeting Including the New Horizons Forum and Aerospace Exposition, American Institute of Aeronautics and Astronautics, Orlando, FL, USA, January 2009.

[11] W. Shyy, H. Aono, S. K. Chimakurthi et al., "Recent progress in flapping wing aerodynamics and aeroelasticity," Recent Progress In Flapping Wing Aerodynamics and Progress in Aerospace Sciences, vol. 46, no. 7, pp. 284-327, 2010.

[12] A. Mohamed, K. Massey, S. Watkins, and R. Clothier, "The attitude control of fixed-wing MAVS in turbulent environments," Progress in Aerospace Sciences, vol. 66, pp. 37-48, 2014.

[13] W. Williams and M. Harris, "The challenges of flight-testing unmanned air vehicles," in Proceedings of the Conference of Systems Engineering, Test and Evaluation, SETE, Brisbane, Australia, May 2002.

[14] J. S. McCarley and C. D. Wickens, Human Factors Concerns in UAV Flight, FAA, Report, Champaign, IL, USA, 2004.

[15] A. Sprater, "Stabilizing Device for Flying Machines," 1119324 , US Patent Office, Alexandria, VA, USA, 1914.

[16] N. F. Harpur, "Effect of Active Control Systems on Structural Design Criteria," Advisory Group for Aerospace Research and Development, AGARD, Washington, DC, USA, 1973.

[17] J. Hawk, R. J. Connor, and C. Levy, Dynamic Analysis of the C-47 Gust Load Alleviation System: SM 14456, Douglas Aircraft, Santa Monica, CA, USA, 1952.

[18] C. C. Kraft, Initial Results of a Flight Investigation of a Gust Alleviation System, NASA, Washington, DC, USA, 1956.

[19] D. McLean, "Gust-alleviation control systems for aircraft," in Proceedings of the Institution of Electrical Engineers-Control and Science, vol. 125, IEE, Loughborough, UK, 1978.

[20] C. J. Blower and A. M. Wickenheiser, "The development of a closed-loop flight controller for localized flow control and gust alleviation using biomimetic feathers on aircraft wings," in Proceedings of the ASME Conference on Smart Materials, Adaptive Structures and Intelligent Systems, Scottsdale, AZ, USA, September 2011.

[21] J. Ratti, M. Jung-Ho, and G. Vachtsevanos, "Towards lowpower, low-profile avionics architecture and control for micro aerial vehicles," Aerospace Conference, vol. 5, pp. 1-8, 2011.

[22] J. K. Shang, S. A. Combes, B. M. Finio, and R. J. Wood, "Artificial insect wings of diverse morphology for flappingwing micro air vehicles," Bioinspiration \& Biomimetics, vol. 4, no. 3, Article ID 036002, 2009.
[23] T. Nakata, H. Liu, Y. Tanaka, N. Nishihashi, X. Wang, and A. Sato, "Aerodynamics of a bio-inspired flexible flappingwing micro air vehicle," Bioinspiration \& Biomimetics, vol. 6, no. 4, Article ID 045002, 2011.

[24] R. P. O'hara and A. N. Palazotto, “The morphological characterization of the forewing of the Manduca sexta species for the application of biomimetic flapping wing micro air vehicles," Bioinspiration \& Biomimetics, vol. 7, no. 4, Article ID 046011, 2012.

[25] G. C. H. E. de Croon, M. A. Groen, C. De Wagter, B. Remes, R. Ruijsink, and B. W. van Oudheusden, "Design, aerodynamics and autonomy of the DelFly," Bioinspiration \& Biomimetics, vol. 7, no. 2, Article ID 025003, 2012.

[26] A. Mohamed, R. Clothier, S. Watkins, R. Sabatini, and M. Abdulrahim, "Fixed-wing MAV attitude stability in atmospheric turbulence PART 1: suitability of conventional sensors," Progress in Aerospace Sciences, vol. 70, 2014.

[27] A. Mohamed, R. Clothier, S. Watkins, R. Sabatini, and R. Abdulrahim, "Fixed-wing MAV attitude stability in atmospheric turbulence PART 2: investigating biologicallyinspired sensors," Progress in Aerospace Sciences, vol. 71, 2014.

[28] Barron Associates, "Adaptive control for synthetic jet actuators," http://www.barron-associates.com/adaptive-controlof-synthetic-jet-actuators/.

[29] C. P. Tilmann, K. J. Langan, J. G. Betterton, and M. J. Wilson, "Characterization of pulsed vortex generator jets for active flow control," in Proceedings of the RTO AVT, Florença, Itália, September 2000.

[30] D. W. Yeo, N. Rehm, J. M. Bradley, and I. Chopra, Flow-Aware Computational Wings for Improved Gust Mitigation on FixedWing Unmanned Aerial Systems, AIAA, San Diego, CA, USA.

[31] N. Moore, "Birds, bats and insects hold secrets for aerospace engineers," http://ns.mich.edu/htdocs/releases/story.php?id=6312.

[32] S. H. Abbasi and A. Mahmood, "Bio-inspired gust mitigation system for a flapping wing UAV: modeling and simulation," Journal of the Brazilian Society of Mechanical Sciences and Engineering, vol. 41, no. 11, p. 524, 2019, https://doi.org/10. 1007/s40430-019-2044-9.

[33] S. H. Abbasi and A. Mahmood, "Modeling, simulation and control of a bio-inspired electromechanical feather for gust mitigation in flapping wing UAV," in Proceedings of the 2019 2nd International Conference on Communication, Computing and Digital systems (C-CODE), pp. 195-200, Islamabad, Pakistan, March 2019.

[34] W. Send, "Artifcial hinged-wing bird with active torsion and partially linear kinematics," in Proceedings of the 28th International Congress of the Aeronautical Sciences, Brisbane, Australia, September 2012.

[35] M. Bucolo, A. Buscarino, L. Fortuna, and S. Gagliano, "Bifurcation scenarios for pilot induced oscillations," Aerospace Science and Technology, vol. 106, Article ID 106194, 2020.

[36] G. Dubois, Taylor, and Francis, Modeling and Simulation, CRC Press, Boca Raton, FL, USA, 2018.

[37] D. C. Karnopp, D. L. Margolis, and R. C. Rosenberg, System Dynamics Modeling and Simulation of Mechatronic Systems, Wiley, Canada, 2000.

[38] L. N. Long and T. E. Fritz, "Object-oriented unsteady vortex lattice method for flapping flight," Journal of Aircraft, vol. 41, no. 6, pp. 1275-1290, 2004.

[39] Z. Jahanbin, A. Selk Ghafari, A. Ebrahimi, and A. Meghdari, "Multi-body simulation of a flapping-wing robot using an efficient dynamical model," Journal of the Brazilian Society of Mechanical Sciences and Engineering, vol. 38, no. 1, pp. 133149, 2016. 
[40] M. Bucolo, A. Buscarino, C. Famoso, L. Fortuna, and M. Frasca, "Control of imperfect dynamical systems," Nonlinear Dynamics, vol. 98, no. 4, pp. 2989-2999, 2019.

[41] S. Karimian and J. Zahra, "Aerodynamic modeling of a flexible flapping-wing micro-air vehicle in the bond graph environment with the aim of assessing the lateral control power," Proceedings of the Institution of Mechanical Engineers Part G: Journal of Aerospace Engineering, vol. 1, 2019. 